\title{
Bioactive steroidal saponins from Agave offoyana flowers
}

\author{
Andy J. Pérez ${ }^{\mathrm{a}, \mathrm{c}}$, Juan M. Calle ${ }^{\mathrm{a}}$, Ana M. Simonet ${ }^{\mathrm{a}}$, José O. Guerra ${ }^{\mathrm{b}}$, Anna Stochmal ${ }^{\mathrm{c}}$, \\ Francisco A. Macías ${ }^{\text {a,* }}$ \\ a Grupo de Alelopatía, Departamento de Química Orgánica, Facultad de Ciencias, Universidad de Cádiz, C/República Saharaui, s/n, 11510 Puerto Real, Cádiz, Spain

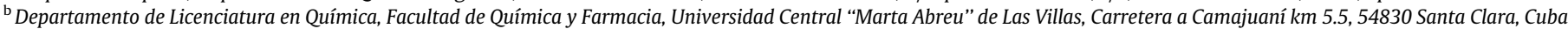 \\ ${ }^{\mathrm{c}}$ Department of Biochemistry, Institute of Soil Science and Plant Cultivation, State Research Institute, ul. Czartoryskich 8, 24-100 Puławy, Poland
}

\section{A R T I C L E I N F O}

\section{Article history}

Received 15 March 2013

Received in revised form 14 June 2013

Available online 13 July 2013

\section{Keywords:}

Agave offoyana

Steroidal saponins

Structural elucidation

Phytotoxic activity

Structure-activity relationship

\begin{abstract}
A B S T R A C T
Bioguided studies of flowers of Agave offoyana allowed the isolation of five steroidal saponins never described previously, Magueyosides A-E (1-5), along with six known steroidal saponins (6-11). The structures of compounds were determined as (25R)-spirost-5-en-2 $\alpha, 3 \beta$-diol-12-one 3-O-\{ $\beta$-D-xylopyranosyl-( $1 \rightarrow 3)-O$ - $\beta$-D-glucopyranosyl-( $1 \rightarrow 2)-O$-[ $\beta$-D-xylopyranosyl-( $1 \rightarrow 3)]-O$ - $\beta$-D-glucopyranosyl-( $1 \rightarrow 4)-O$ - $\beta$-D-galactopyranoside $\}$ (1), (25R)-spirost-5-en-2 $\alpha, 3 \beta$-diol-12-one 3-O-\{ $\beta$-D-glucopyranosyl-( $1 \rightarrow 2)-O$-[ $\beta$-D-xylopyranosyl- $(1 \rightarrow 3)]-O$ - $\beta$-D-glucopyranosyl- $(1 \rightarrow 4)-O$ - $\beta$-D-galactopyranoside $\}$ (2), (25R)-spirost-5-en-2 $\alpha, 3 \beta, 12 \beta$-triol 3 - $O$ - $\{\beta$-D-glucopyranosyl- $(1 \rightarrow 2)-O$-[ $\beta$-D-xylopyranosyl- $(1 \rightarrow 3)]$ $O$ - $\beta$-D-glucopyranosyl- $(1 \rightarrow 4)$ - $O$ - $\beta$-D-galactopyranoside $) \quad(3), \quad(25 R)-5 \alpha$-spirostan- $2 \alpha, 3 \beta$-diol- 12 -one 3-O- $\{\beta$-D-xylopyranosyl-( $1 \rightarrow 3)-O$ - $\beta$-D-glucopyranosyl- $(1 \rightarrow 2)-O$-[ $\beta$-D-xylopyranosyl-( $1 \rightarrow 3)]-O-\beta$-D-glucopyranosyl-( $1 \rightarrow 4)-O-\beta$-D-galactopyranoside $\}$ (4), and (25R)-5 $\alpha$-spirostan-2 $\alpha, 3 \beta$-diol-9(11)-en-12-one 3-O-\{ $\beta$-D-xylopyranosyl-( $1 \rightarrow 3)-O$ - $\beta$-D-glucopyranosyl- $(1 \rightarrow 2)-O$-[ $\beta$-D-xylopyranosyl- $(1 \rightarrow 3)]-O-\beta$-D-glucopyranosyl-( $(1 \rightarrow 4)-O-\beta$-D-galactopyranoside $\}(\mathbf{5})$, by comprehensive spectroscopic analysis, including one- and two-dimensional NMR techniques, mass spectrometry and chemical methods. The bioactivities of the isolated compounds on the standard target species Lactuca sativa were evaluated. A dosedependent phytotoxicity and low dose stimulation were observed.
\end{abstract}

(c) 2013 Elsevier Ltd. All rights reserved.

\section{Introduction}

The Agavaceae family includes more than 480 species that occur natively in America, which are distributed among United States, Central America and Antilles, with larger diversification in Mexico. Those plants are present in Cuba with several genera, in the case of Agave genus, with around 16 species with an even distribution throughout Cuba, which are popularly known as "Maguey". One of them is Agave offoyana, which grows as endemic plant in the central region (Álvarez de Zayas, 1996).

Many species from Agave genus have been extensively investigated in chemical constituents, such as A. ghiesbrechtii (Blunden et al., 1974), A. cantala (Varshney et al., 1981, 1982; Sharma and Sati, 1982; Pant et al., 1986; Sati et al., 1985, 1987; Jain, 1987; Uniyal et al., 1990, 1991a,b), A. sisalana (Blunden et al., 1986; Ding et al., 1989, 1993; Zou et al., 2006; Chen et al., 2009, 2011), A. utahensis (Yokosuka and Mimaki, 2007, 2009), A. shrevei (Pereira da Silva et al., 2006a), A. americana (Jin et al., 2002a,b, 2003b, 2004; Jin and Yang, 2003a; Yokosuka et al., 2000; Tinto et al., 2005), A. fourcroydes (Ohtsuki et al., 2004), A. attenuata Salm-Dyck

\footnotetext{
* Corresponding author. Tel.: +34 956 012770; fax: +34 956016193.

E-mail address: famacias@uca.es (F.A. Macías).
}

(Paz Mendes et al., 2004; Pereira da Silva et al., 2002), A. macroacantha Zucc (Eskander et al., 2010), A. dicipiens Baker (Abdel Gawad et al., 1999), A. brittoniana Trel. Spp Brachypus (Macías et al., 2007, 2010), A. barbadensis (Tinto et al., 2005) and A. tequilana Weber (Morales Serna et al., 2010). However, phytochemical studies of A. offoyana have not been reported so far.

The main secondary metabolites isolated from leaves, roots and flowers are steroidal saponins, to which various biological properties (Sparg et al., 2004), such as hemolytic (Paz Mendes et al., 2004; Pereira da Silva et al., 2006b), molluscicide (Pant et al., 1986; Abdel Gawad et al., 1999), anti-inflammatory (Pereira da Silva et al., 2002), antifungal (Yang et al., 2006; Zhang et al., 2008), anti-bacterial (Killeen et al., 1998; Qin et al., 2012); antiplatelet aggregation (Li et al., 2010; Kang et al., 2012) and cytotoxic (Itabashi et al., 2000; Yokosuka and Mimaki, 2009; Ohtsuki et al., 2004; Mimaki et al., 1998, 2000) have been attributed. However, few studies reporting phytotoxicity of these metabolites have been described, concerning usually the triterpenoid saponins.

A bioguided isolation of the phytotoxic constituents of flowers of $A$. offoyana was performed. The isolation and structural elucidation of five steroidal saponins, named Magueyosides A-E (1-5), along with six known steroidal saponins, YS-IX (6) (Nakano et al., 1991), Agabrittonoside A (7) (Macías et al., 2007), Karatavioside 
A (8) (Vollermer et al., 1978), 3-O-[ $\beta$-D-glucopyranosyl]-6-O-[ $\beta$-Dglucopyranosyl] chlorogenin (9) (Sharma and Sati, 1982), Hecogenin 3-O- $\{\beta$-D-xylopyranosyl- $(1 \rightarrow 3)-O-\beta$-D-glucopyranosyl$(1 \rightarrow 2)-O$-[ $\beta$-D-xylopyranosyl-( $1 \rightarrow 3)]-O-\beta$-D-glucopyranosyl- $(1 \rightarrow 4)$ $O$ - $\beta$-D-galactopyranoside (10) (Mimaki et al., 2000) and Hecogenin 3-O-\{ $\beta$-D-glucopyranosyl- $(1 \rightarrow 2)-O$-[ $\beta$-D-xylopyranosyl- $(1 \rightarrow 3)]-O$ $\beta$-D-glucopyranosyl-( $1 \rightarrow 4)-O$ - $\beta$-D-galactopyranoside\} (11) (Mimaki et al., 1995) is presented in this work.

This is the first time that Agabrittonoside $A$ as a pure compound is obtained; its optical rotation and HRMSFAB are described. Phytotoxic activity of the isolated compounds against Lactuca sativa $\mathrm{L}$. was evaluated.

\section{Results and discussion}

\subsection{Characterization of the compounds}

Dried flowers of $A$. offoyana were extracted exhaustively with EtOH- $\mathrm{H}_{2} \mathrm{O}$ (7:3). This extract was partitioned ( $n$-BuOH-water), and the $n-\mathrm{BuOH}$-soluble portion was subjected to a bioassayguided fractionation by VLC using RP-18 as stationary phase to give five fractions. Obtained extracts and fractions were assayed on etiolated wheat coleoptiles (Macías et al., 2000) at 800, 400, 200 ppm (Fig. 1). A high inhibitory activity of the extracts was observed, which after its fractionation appeared in $\mathrm{D}$ and $\mathrm{E}$ fractions.

These fractions (D and E) were selected for the phytotoxic evaluation (Fig. 2). The concentrations tested were identical to those for the previous bioassay. Standard target species (STS) were L. sativa L. (lettuce), Lycopersicum esculentum Will. (tomato), Lepidium sativum L. (cress), and Allium cepa L. (onion). The tested fractions did not cause significant effects on the germination of the evaluated seeds, unlike root growth of the seeds, which was highly inhibited. Higher than $60 \%$ of inhibitory effect on roots growth of L. sativa, L. sativum and $A$. cepa, at $800 \mathrm{ppm}$ of fraction D was shown. These activity values were greater than those of the herbicide Logran ${ }^{\circledR}$ on lettuce and cress. The shoots growth was slightly affected, only a $40 \%$ of inhibition on L. sativa, L. sativum and A. cepa for the higher concentration of fraction $\mathrm{D}$ was observed as the best result.

After multiple separation processes by normal and reverse phase medium-pressure liquid chromatography (MPLC) and HPLC of D and E fractions, eleven saponins 1-11 were obtained (Fig. 3). Their structures were elucidated on the basis of spectroscopy data using ${ }^{1} \mathrm{H}$ and ${ }^{13} \mathrm{C}$ NMR, 2D (HSQC, HMBC, DQF-COSY, TOCSY, ROESY, HSQC-TOCSY), selective excitation 1D-ROESY and 1D-TOCSY acquired "in array" experiments, as well as HRFABMS and acid hydrolysis.

Compound 1 (Magueyoside A), a white amorphous solid, exhibited in the HR-FAB-MS (positive ion mode) a quasi-molecular ion

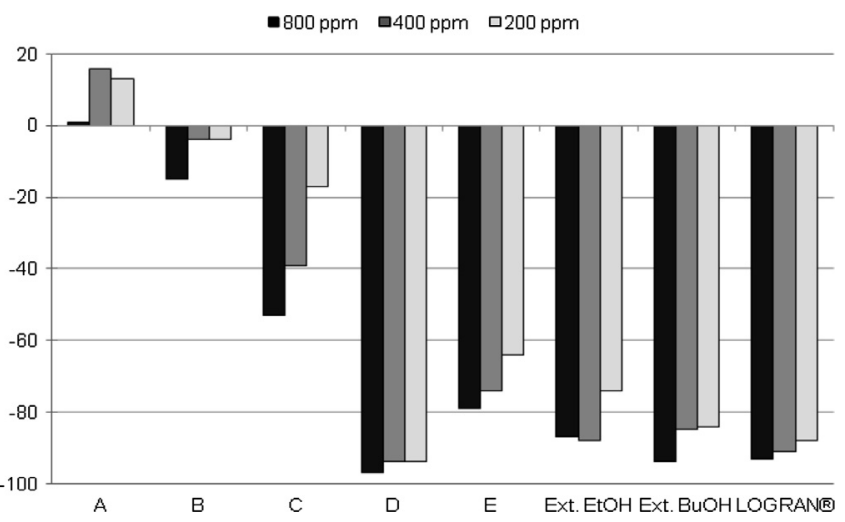

Fig. 1. Effect of extracts and fractions of Agave offoyana flowers on the etiolated wheat coleoptile elongation. Values are expressed as percentage from the control. peak at $m / z$ 1217.5201 [M+Na $]^{+}$(calcd. 1217.5203), which was consistent with the molecular formula of $\mathrm{C}_{55} \mathrm{H}_{86} \mathrm{O}_{28}$. NMR data of compound 1 (Tables 1 and 2) showed similar characteristics as those in Agabrittonoside A (7), a previously described saponin from the same genus (Macías et al., 2007), which was also isolated in this work in pure form. ${ }^{1} \mathrm{H}$ and ${ }^{13} \mathrm{C}$ chemical shifts of sugars portion showed a great similitude with those reported for compound $\mathbf{7}$, as well as in the connectivity between sugar units determined by HMBC and ROESY correlations between linkage points. This suggested the same sugar chain. Absolute configurations of sugar units were determined using the method reported by Tanaka et al., 2007 with slight modification. Compound $\mathbf{1}$ was hydrolyzed and converted into the thiazolizine derivatives to arylthiocarbamate using L-cystein methyl ester and o-tolylisothiocyanate. Then, the reaction mixture was directly analyzed by C-18 HPLC and the retentions time $(R t)$ were compared with values obtained for derivatives of each D- or L- authentic sugars. The peaks at $12.59,14.63$ and 17.60 min coincided with derivative of $D$-galactose, $D$-glucose and D-xylose (Rt of D-galactose: 12.63, Rt of D-glucose: 14.57, Rt of D-xylose: 17.69), respectively.

On the other hand, significant differences in the NMR data of $C$ and $\mathrm{D}$ rings for the aglycone moieties were observed. A signal at $\delta 212.4$ in the ${ }^{13} \mathrm{C}$ NMR spectrum suggested the presence of a carbonyl group, which showed HMBC correlations with protons at $\delta 2.52$ and $2.37(2 \mathrm{H}-11), 2.77(\mathrm{H}-17)$ and $1.06(\mathrm{H}-18)$, consistent with its location in $\mathrm{C}-12$. This was confirmed by the analysis of the coupling constants of $2 \mathrm{H}-11$ signals; $\delta 2.52(\mathrm{dd}, J=14.8,12.7 \mathrm{~Hz})$ and $\delta 2.37$ (dd, $J=14.8,5.8 \mathrm{~Hz}$ ), which shown only one vicinal coupling $\left({ }^{3} \mathrm{~J}\right)$ with $\mathrm{H}-9$.

Finally, the correlations in 2D-ROESY were studied and the aglycone of 1 was elucidated as (25R)-spirost-5-en-2 $\alpha, 3 \beta$-diol-12-one, which has been reported previously as Kammogenin (Marker et al., 1943).

The connection between sugars chain and aglycone was established to be at $\mathrm{C}-3$, by means of HMBC cross-peaks between $\mathrm{H}-1_{\text {gal }}$ $(\delta 4.89)$ and C-3 ( $\delta$ 84.2) of Kammogenin, as well as between $\mathrm{H}-3(\delta$ 3.78) of Kammogenin and $\mathrm{C}-1_{\text {gal }}(\delta 103.2)$. This was confirmed by a NOE correlation of $\mathrm{H}-1_{\text {gal }}$ with $\mathrm{H}-3$ of the aglycone. Therefore, the structure of 1 was established as Kammogenin-3-O-\{ $\beta$-D-Xylopyranosyl-( $1 \rightarrow 3)-O$ - $\beta$-D-glucopyranosyl- $(1 \rightarrow 2)-O$-[ $\beta$-D-xylopyranosyl$(1 \rightarrow 3)]-O$ - $\beta$-D-glucopyranosyl-( $1 \rightarrow 4)-O$ - $\beta$-D-galactopyranoside $\}$. This compound has not been previously described and we propose its name as Magueyoside A.

Compound 2 (Magueyoside $\mathrm{B}$ ) was isolated as a white amorphous solid, with molecular formula $\mathrm{C}_{50} \mathrm{H}_{78} \mathrm{O}_{24}$ deduced by $\mathrm{HR}-$ FAB-MS, which showed in the positive mode a quasi-molecular ion peak at $m / z 1085.4830[\mathrm{M}+\mathrm{Na}]^{+}$, calculated for $\mathrm{C}_{50} \mathrm{H}_{78} \mathrm{O}_{24} \mathrm{Na}$, 1085.4781. ${ }^{13} \mathrm{C}$ and ${ }^{1} \mathrm{H}$ NMR characteristics of the aglycone of compound $\mathbf{2}$ (Table 1 ) were similar to those of compound $\mathbf{1}$. However, differences in sugars portion were observed. The ${ }^{1} \mathrm{H}$ NMR spectrum showed only four signals of anomeric protons at $\delta 4.89,5.18,5.56$ and 5.22, which showed HSQC correlations with $\delta$ 103.2, 104.6, 104.7 and 104.9 respectively. Differences of chemical shifts between compounds $\mathbf{1}$ and 2, mainly for the second glucose unit $\left(\mathrm{Glc}^{\prime}\right)$, were observed (Table 2). The signal of $\mathrm{C}-3_{\mathrm{Glc}^{\prime}}$ in compound $\mathbf{2}$ was assigned at $\delta 78.0$, while this in compound $\mathbf{1}$ is at $\delta 87.0$, which suggest the lack of a glycosidic bond in this carbon of compound 2. Therefore, the same sugars chain of compound $\mathbf{1}$, but without a xylose unit was suggested. The absolute configurations of sugar units were determined in the same way employed in compound 1. The peaks at $12.59,14.63$ and 17.60 min coincided with derivatives of D-galactose, D-glucose and D-xylose (Rt of D-galactose: 12.63, Rt of D-glucose: 14.57, Rt of D-xylose: 17.69), respectively. Thus, the compound $\mathbf{2}$ was elucidated as Kammogenin$3-O$ - $\{\beta$-D-glucopyranosyl-( $1 \rightarrow 2)-O$-[ $\beta$-D-xylopyranosyl- $(1 \rightarrow 3)]-O-\beta$ D-glucopyranosyl-( $1 \rightarrow 4)-O-\beta$-D-galactopyranoside $\}$. This compound 

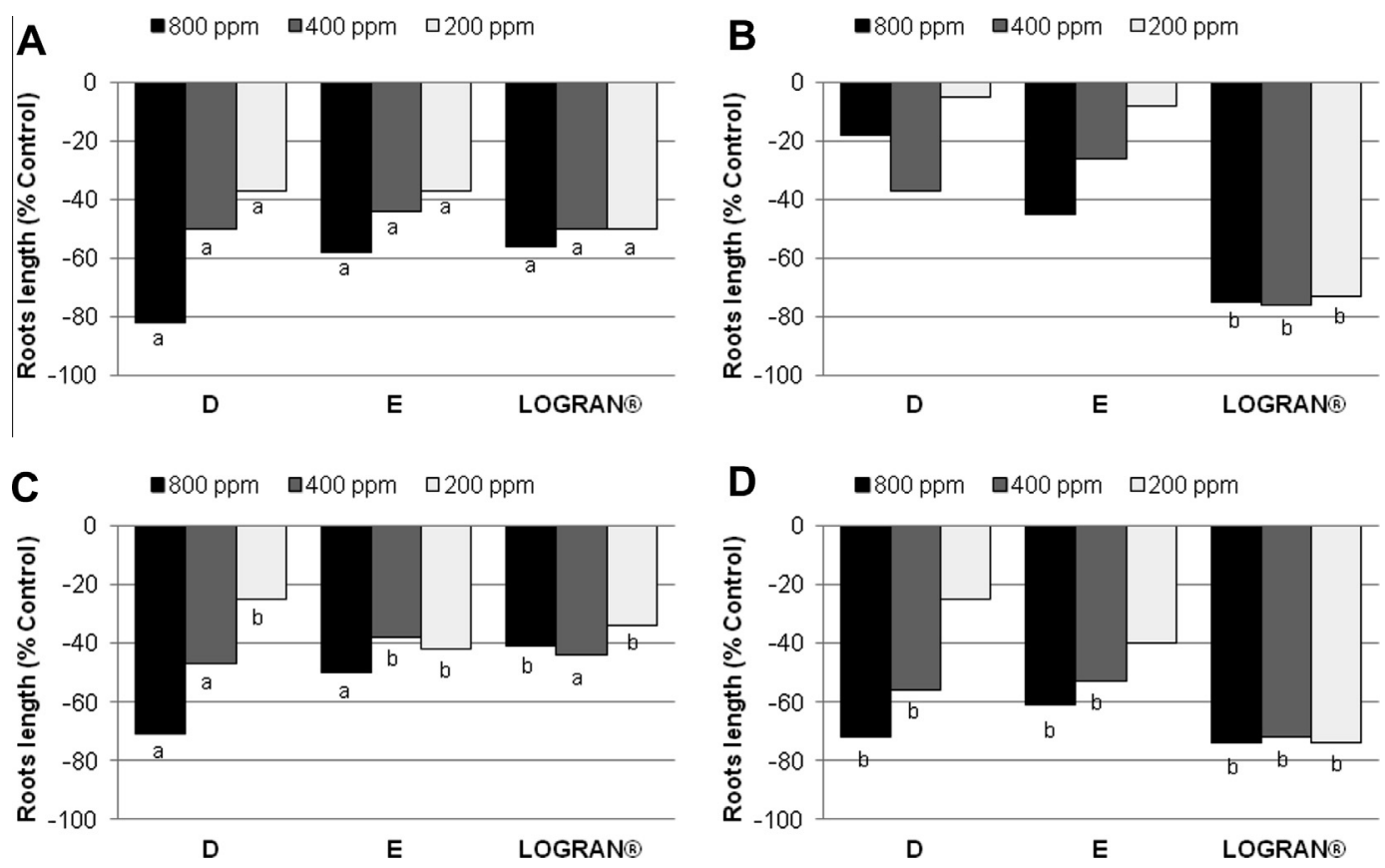

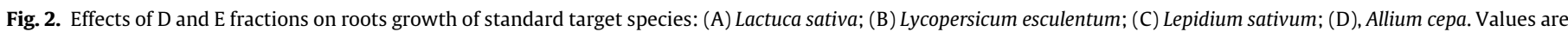

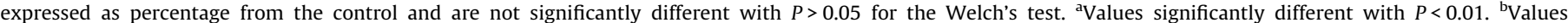
significantly different with $0.01<P<0.05$.

has not been previously described and represents a new natural steroidal saponin with proposed name as Magueyoside B.

Compound 3 (Magueyoside $\mathrm{C}$ ) was isolated as a white amorphous solid; the molecular formula was determined as $\mathrm{C}_{50} \mathrm{H}_{80} \mathrm{O}_{24}$ (HR-FAB-MS: $m / z 1087.4919[\mathrm{M}+\mathrm{Na}]^{+}$, calculated for $\mathrm{C}_{50} \mathrm{H}_{80} \mathrm{O}_{24} \mathrm{Na}$, 1087.4937). This indicated two mass units more than compound 2. Its characteristics in ${ }^{13} \mathrm{C}$ and ${ }^{1} \mathrm{H}$ NMR spectra for the sugars portion (Table 2) were in agreement with those of $\mathbf{2}$, and the exhaustive studies of HMBC and 1D-ROESY spectra confirmed the same connectivity. The absolute configuration of all sugars were determined as $D$, by means of the same method described above. However, some differences of the resonance signals in the $C$ and $D$ rings of the aglycones of $\mathbf{3}$ and $\mathbf{2}$ were observed (Table 1), as for example the absence of the carbonyl group signal corresponding to C-12 in ${ }^{13} \mathrm{C}$ NMR spectrum of 3 . Nevertheless, the HSQC spectrum showed the presence of methine group by the cross peak between a signal at $\delta_{\mathrm{H}} 3.52$ and $\delta_{\mathrm{C}} 78.8$. These signals were assigned as $\mathrm{H}-12$ and $\mathrm{C}-12$ respectively, due to the HMBC correlations of $\delta_{\mathrm{C}} 78.8$ with methyl-18 $(\delta 1.06)$ and the signals at $\delta 1.67$ and $\delta 1.93$ (2H-11). So, the presence of a hydroxyl group at C-12 of the aglycone of $\mathbf{3}$ was suggested. The values of coupling constants of $\mathrm{H}-12, \delta 3.52 \mathrm{dd}$ $(11.1,4.7 \mathrm{~Hz})$, were consistent with the vicinal coupling ${ }^{3} \mathrm{Jax}$-ax and ${ }^{3} J_{a x-e q}$, which displayed an equatorial position for hydroxyl group in $\mathrm{C}-12$.

The correlations in 2D-ROESY for the aglycone of $\mathbf{3}$ were finally studied (Fig. 4). Cross peaks of $\mathrm{H}-12(\delta 3.52)$ with $\mathrm{H}-11_{\text {eq }}(\delta 1.93)$, $\mathrm{H}-14(\delta 1.07), \mathrm{H}-9(\delta 1.10)$ and $\mathrm{H}-17(\delta 2.15)$ confirmed an axial disposition of $\mathrm{H}-12$ due to that all of them are located to the " $\alpha$ " plane. Furthermore, signal of methyl-19 $(\delta$ 0.95) showed correlations with the protons at $\delta 4.00(\mathrm{H}-2), 2.51\left(\mathrm{H}_{\mathrm{ax}}-4\right), 2.29\left(\mathrm{H}_{\mathrm{eq}}-1\right)$, $1.51(\mathrm{H}-8)$ and $1.67\left(\mathrm{H}_{\mathrm{ax}}-11\right)$, the latter two were commons for methyl-18, which indicated a "trans" junction of A and B rings, as well as " $\beta$ " orientation for both methyl groups. Also, the relative configurations of other important chirals carbons in the aglycone were established. Shown correlations between methyl-21 $(\delta 1.41)$ and the protons at $\delta 2.15(\mathrm{H}-17)$ and $\delta 4.60(\mathrm{H}-16)$, allowed determine a " $\alpha$ " orientation for this methyl group, which is verified by the H-20 ( $\delta 2.16)$ and methyl-18 ( $\delta$ 1.06) cross peak. This, enables establish the typical "cis" junction between D and E rings and the "S" configuration for C-20. Similarly, the ketal carbon C-22 was determined as "R", due to the ROESY correlation between $\mathrm{H}_{\mathrm{ax}}-26$ $(\delta 3.52)$ and $\mathrm{H}-16(\delta 4.60)$, as well as between $\mathrm{H}-20(\delta 2.16)$ and $\mathrm{H}_{2}-23(\delta 1.68,1.71)$. Other correlations, such as $\mathrm{H}_{\mathrm{ax}}-7 / \mathrm{H}-9, \mathrm{H}_{\mathrm{ax}}-7 /$ $\mathrm{H}-14$ and $\mathrm{H}-14 / \mathrm{H}-16$, allowed complete the relative stereochemistry of aglycone of $\mathbf{3}$ with the usual B/C "trans" and C/D "trans" junctions.

Thus, the aglycone of 3 was elucidated as (25R)-spirost-5en-2 $\alpha, 3 \beta, 12 \beta$-triol and is reported here for first time as a natural steroid. Finally, the structure of $\mathbf{3}$ was established as (25R)-spirost-5-en- $2 \alpha, 3 \beta, 12 \beta$-triol 3 -O- $\{\beta$-D-glucopyranosyl- $(1 \rightarrow 2)-O$-[ $\beta$-Dxylopyranosyl-( $1 \rightarrow 3)]-O$ - $\beta$-D-glucopyranosyl- $(1 \rightarrow 4)-O$ - $\beta$-D-galactopyranoside\}. This compound has not been described before and its name was proposed as Magueyoside $C$.

Compound 4 (Magueyoside D), was a white amorphous solid, with a molecular formula $\mathrm{C}_{55} \mathrm{H}_{88} \mathrm{O}_{28}$ (HR-FAB-MS: $\mathrm{m} / z 1219.5406$ $[\mathrm{M}+\mathrm{Na}]^{+}$, calculated for $\left.\mathrm{C}_{55} \mathrm{H}_{88} \mathrm{O}_{28} \mathrm{Na}, 1219.5360\right)$. Its ${ }^{1} \mathrm{H}$ and ${ }^{13} \mathrm{C}$ NMR data (Tables 1 and 2) were in good agreement with those previously described for YS-IX (6) (Nakano et al., 1991), but with variations in the sugars chain. The same chain showed by Magueyoside A (1) and Agabrittonoside A (7), with five monosaccharide moieties, was determined to be also in compound 4. After acid hydrolysis all of sugar units were identified as D. Consequently, the structure of compound $\mathbf{4}$ was established as Manogenin-3-O-\{ $\beta$-D-Xylopyranosyl- $(1 \rightarrow 3)-O-\beta$-D-glucopyranosyl$(1 \rightarrow 2)-O$-[ $\beta$-D-Xylopyranosyl- $(1 \rightarrow 3)]-O$ - $\beta$-D-glucopyranosyl- $(1 \rightarrow 4)$ $O$ - $\beta$-D-galactopyranoside , which has not been described before and the proposed name is Magueyoside D.

Compound 5 (Magueyoside E) was a white amorphous solid, with the same molecular formula $\left(\mathrm{C}_{55} \mathrm{H}_{86} \mathrm{O}_{28}\right.$, HR-FAB-MS: $\mathrm{m} / z$ 1217.5211 $[\mathrm{M}+\mathrm{Na}]^{+}$, calculated for $\left.\mathrm{C}_{55} \mathrm{H}_{86} \mathrm{O}_{28} \mathrm{Na}, 1217.5203\right)$ as compound 1. The NMR features of the sugars portions (Table 2) were similar of those of compound $\mathbf{1}$, indicating the same sugars chain. The $\mathrm{D}$ series of all sugar moieties was determined in the same way described above. However, important differences in 

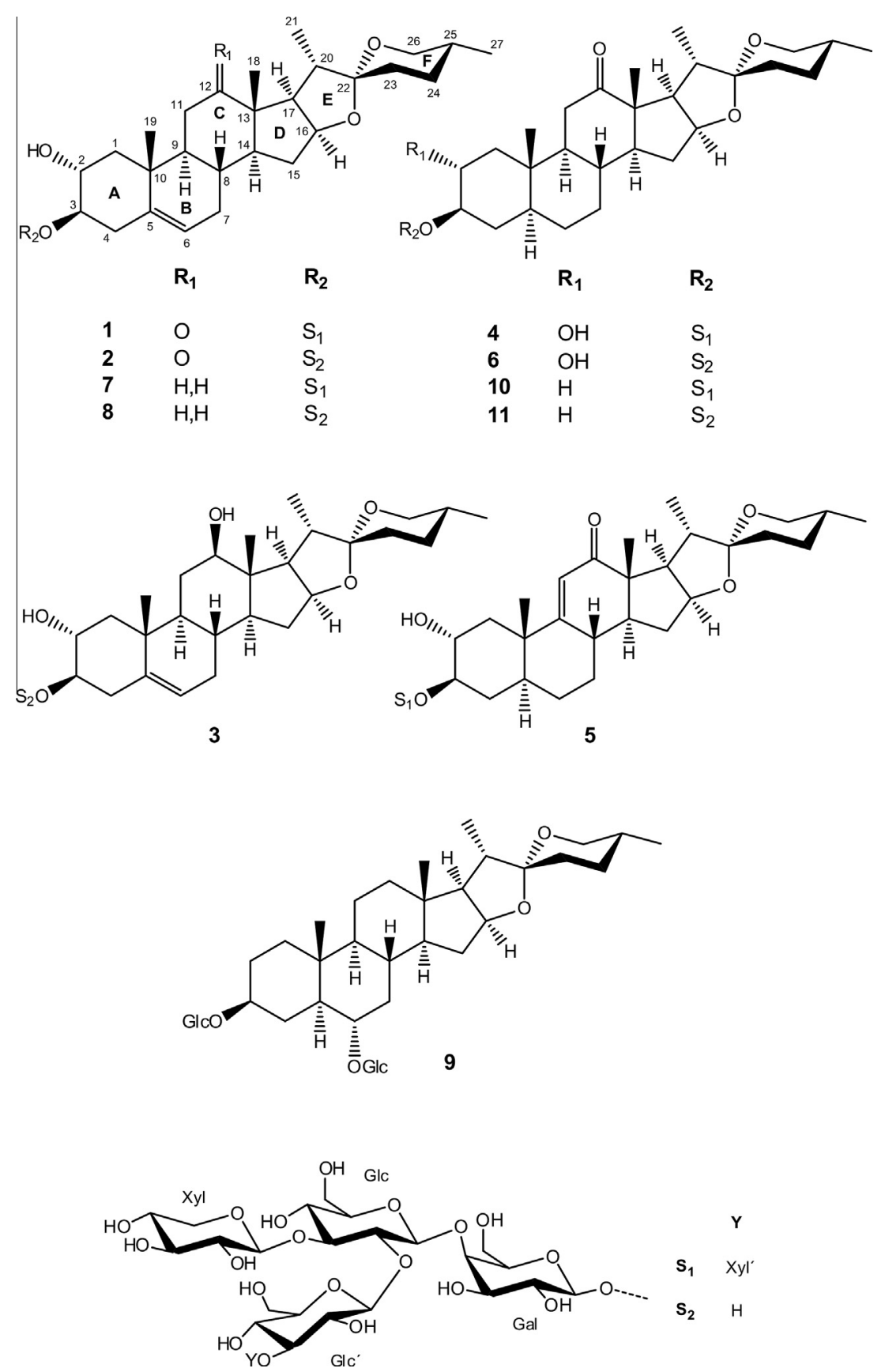

Fig. 3. Chemical structures of saponins 1-11, isolated from flowers of $A$. offoyana.

their aglycones, especially into the A-C rings were detected. ${ }^{13} \mathrm{C}$ NMR spectrum of $\mathbf{5}$ (Table 1 ) showed a resonance signal of the carbonyl group at $\delta 204.2$, which is shifted to higher field than that of 1. As in compounds 1,2 and 4 , this was assigned in C-12 by means of HMBC correlations through three bonds with methyl group at C-18 $(\delta 0.97)$ and $\mathrm{H}-17(\delta 2.61)$. Moreover, the double bond was found between $\mathrm{C}-9$ and $\mathrm{C}-11$ instead of between $\mathrm{C}-5$ and C- 6 , due to the HMBC cross-peaks of proton at $\delta 5.93$ with C-8 ( $\delta$ 36.1), C-10 ( $\delta$ 40.5) and C-13 ( $\delta$ 51.3). Thus, the occurrence of $\alpha, \beta$-unsaturated carbonyl group was suggested and the structure of the aglycone moiety of $\mathbf{5}$ was established to be 9-dehydromanogenin (Mimaki et al., 1995). Finally, the structure of $\mathbf{5}$ was elucidated as 9-dehydromanogenin-3-O- $\{\beta$-D-xylopyranosyl$(1 \rightarrow 3)-O$ - $\beta$-D-glucopyranosyl- $(1 \rightarrow 2)-O$-[ $\beta$-D-xylopyranosyl- $(1 \rightarrow 3)]-$ $O$ - $\beta$-D-glucopyranosyl-( $1 \rightarrow 4)-O$ - $\beta$-D-galactopyranoside . This com- pound has not been described before and the proposed name is Magueyoside E.

\subsection{Bioactivity}

There is low number of precedents of phytotoxicity studies of saponins. Mainly oleanane saponins are assayed (Waller et al., 1996; Oleszek, 1993; Tsurumi and Tsujino, 1995; Hernández Carlos et al., 2011; Scognamiglio et al., 2012).

Looking for the physiological role of steroids in the higher plants, some authors used steroidal saponins, such as digitonin (Vendrig, 1964) or diosgenin derivatives (Helmkamp and Bornner, 1953) finding a growth promotion on avena section tests or embryos of mature pea seeds. 
Table 1

${ }^{13} \mathrm{C}$ and ${ }^{1} \mathrm{H}$ NMR data $\left(\mathrm{J}\right.$ in $\mathrm{Hz}$ ) of the aglycone moieties of compounds $\mathbf{1 - 5}$ (pyridine- $\mathrm{d}_{5}, 600 \mathrm{MHz}$ ).

\begin{tabular}{|c|c|c|c|c|c|c|c|c|c|c|}
\hline & \multicolumn{2}{|c|}{ Magueyoside A (1) } & \multicolumn{2}{|c|}{ Magueyoside B (2) } & \multicolumn{2}{|c|}{ Magueyoside C (3) } & \multicolumn{2}{|c|}{ Magueyoside D (4) } & \multicolumn{2}{|c|}{ Magueyoside E (5) } \\
\hline & $\delta_{\mathrm{C}}$ & $\delta_{\mathrm{H}}$ & $\delta_{\mathrm{C}}$ & $\delta_{\mathrm{H}}$ & $\delta_{\mathrm{C}}$ & $\delta_{\mathrm{H}}$ & $\delta_{\mathrm{C}}$ & $\delta_{\mathrm{H}}$ & $\delta_{\mathrm{C}}$ & $\delta_{\mathrm{H}}$ \\
\hline $1_{\mathrm{ax}}$ & 45.2 & $1.22 \mathrm{dd}(12.4,12.9)$ & 45.1 & $1.21 \mathrm{dd}(12.3,12.8)$ & 45.7 & $1.28 \mathrm{dd}(12.2,12.9)$ & 45.0 & $1.09 \mathrm{~m}$ & 43.4 & $1.55 \mathrm{dd}(12.7,10.9)$ \\
\hline $1_{\mathrm{eq}}$ & & $2.14 \mathrm{dd}(12.9,4.5)$ & & $2.13 \mathrm{dd}(12.8,4.5)$ & & $2.29 \mathrm{dd}(12.9,4.7)$ & & $2.00 \mathrm{dd}(12.6,4.7)$ & & $2.21 \mathrm{dd}(12.7,4.8)$ \\
\hline 2 & 69.7 & $3.99 \mathrm{~m}$ & 69.7 & $3.99 \mathrm{~m}$ & 69.9 & $4.00 \mathrm{~m}$ & 70.1 & $3.89 \mathrm{~m}$ & 70.2 & $4.00 \mathrm{~m}$ \\
\hline 3 & 84.2 & $3.78 \mathrm{ddd}(11.4,8.9,5.2)$ & 84.0 & $3.78 \mathrm{ddd}(11.9,9.1,5.1)$ & 84.5 & 3.79 ddd $(11.5,8.9,5.2)$ & 83.9 & 3.82 ddd $(10.6,8.9,4.9)$ & 83.4 & $3.83 \mathrm{ddd}(11.0,8.9,4.9)$ \\
\hline $4_{\mathrm{ax}}$ & 37.4 & $2.51 \mathrm{dd}(14.0,11.4)$ & 37.4 & $2.49 \mathrm{dd}(14.0,11.9)$ & 37.5 & $2.51 \mathrm{dd}(13.9,11.5)$ & 33.8 & 1.44 ddd $(13.2,11.9,10.6)$ & 33.6 & $1.49 \mathrm{ddd}(13.2,12.8,11.0)$ \\
\hline $4_{\mathrm{eq}}$ & & $2.71 \mathrm{dd}(14.0 .5 .2)$ & & $2.70 \mathrm{dd}(14.0,5.1)$ & & $2.69 \mathrm{dd}(13.9,5.3)$ & & 1.84 ddd $(13.2,4.9,2.8)$ & & $1.91 \mathrm{ddd}(13.2,5.3,3.0)$ \\
\hline 5 & 139.8 & - & 139.8 & - & 140.0 & - & 44.3 & $0.93 \mathrm{~m}$ & 42.4 & $1.15 \operatorname{tdd}(12.8,13.0,3.0,3.3)$ \\
\hline $6_{\mathrm{ax}}$ & 121.6 & $5.25 \mathrm{dd}(5.2,2.3)$ & 121.6 & $5.25 \mathrm{dd}(5.1,2.4)$ & 122.0 & $5.28 \mathrm{dd}(5.7,2.7)$ & 27.7 & $1.03 \mathrm{~m}$ & 27.1 & $1.23 \mathrm{dq}(13.0,3.7)$ \\
\hline $6_{\text {eq }}$ & & & & & & & & $1.12 \mathrm{~m}$ & & $1.16 \mathrm{~m}$ \\
\hline $7_{\mathrm{ax}}$ & 31.6 & $1.43 \mathrm{~m}$ & 31.6 & $1.42 \mathrm{~m}$ & 32.0 & $1.43 \mathrm{~m}$ & 31.5 & $0.72 \mathrm{dq}(12.7,4.7)$ & 32.4 & $0.87 \mathrm{~m}$ \\
\hline $7_{\text {eq }}$ & & $1.84 \mathrm{~m}$ & & $1.83 \mathrm{~m}$ & & $1.83 \mathrm{ddd}(12.1,4.8,2.7)$ & & $1.50 \mathrm{~m}$ & & $1.73 \mathrm{~m}$ \\
\hline 8 & 30.3 & $1.78 \mathrm{~m}$ & 30.3 & $1.78 \mathrm{~m}$ & 30.3 & $1.51 \mathrm{dq}(4.8,10.8,10.7,10.7)$ & 33.6 & $1.70 \mathrm{dq}(12.7,4.3)$ & 36.1 & $2.36 \operatorname{tdd}(10.7,10.7,5.7,2.1)$ \\
\hline 9 & 52.0 & $1.36 \mathrm{~m}$ & 52.0 & $1.34 \mathrm{~m}$ & 49.9 & 1.10 ddd $(10.8,10.7,4.7)$ & 55.3 & $0.96 \mathrm{~m}$ & 170.4 & - \\
\hline 10 & 38.3 & - & 38.3 & - & 38.0 & - & 38.0 & - & 40.5 & - \\
\hline $11_{\mathrm{ax}}$ & 37.4 & $2.52 \mathrm{dd}(14.8,12.7)$ & 37.4 & $2.51 \mathrm{dd}(14.6,12.9)$ & 31.5 & $1.67 \mathrm{~m}$ & 37.2 & $2.38 \mathrm{dd}(14.3,13.6)$ & 120.1 & $5.93 \mathrm{~d}(2.1)$ \\
\hline $11_{\text {eq }}$ & & $2.37 \mathrm{dd}(14.8,5.8)$ & & $2.35 \mathrm{dd}(14.6,5.8)$ & & $1.93 \mathrm{ddd}(13.1,4.7,4.7)$ & & $2.32 \mathrm{dd}(14.3,5.2)$ & & \\
\hline 12 & 212.4 & - & 212.4 & - & 78.8 & $3.52 \mathrm{dd}(11.1,4.7)$ & 212.5 & - & 204.2 & - \\
\hline 13 & 54.9 & - & 54.9 & - & 46.2 & - & 55.3 & - & 51.3 & - \\
\hline 14 & 55.7 & $1.40 \mathrm{~m}$ & 55.7 & $1.37 \mathrm{~m}$ & 55.2 & $1.07 \mathrm{~m}$ & 55.7 & $1.33 \mathrm{~m}$ & 52.6 & $1.69 \mathrm{~m}$ \\
\hline $15_{\mathrm{ax}}$ & 31.5 & $1.58 \mathrm{~m}$ & 31.5 & $1.57 \mathrm{~m}$ & 31.9 & $1.56 \mathrm{~m}$ & 31.4 & $1.55 \mathrm{~m}$ & 31.8 & $1.62 \mathrm{~m}$ \\
\hline $15_{\mathrm{eq}}$ & & $2.07 \mathrm{ddd}(12.1,6.8,5.3)$ & & $2.06 \mathrm{ddd}(12.1,7.2,5.9)$ & & 2.05 ddd $(11.7,6.7,5.3)$ & & $2.07 \mathrm{ddd}(11.6,6.7,5.7)$ & & $2.16 \mathrm{ddd}(11.4,7.1,4.8)$ \\
\hline 16 & 79.7 & $4.45 \mathrm{~m}$ & 79.6 & $4.44 \mathrm{~m}$ & 81.1 & $4.60 \mathrm{~m}$ & 79.6 & $4.45 \mathrm{~m}$ & 80.2 & $4.50 \mathrm{~m}$ \\
\hline 17 & 53.9 & $2.77 \mathrm{dd}(8.9,6.6)$ & 53.9 & $2.76 \mathrm{dd}(8.7,6.8)$ & 62.7 & $2.15 \mathrm{~m}$ & 54.2 & $2.73 \mathrm{dd}(8.6,6.7)$ & 54.5 & $2.61 \mathrm{dd}(8.8,7.1)$ \\
\hline 18 & 15.8 & $1.06 \mathrm{~s}$ & 15.8 & $1.05 \mathrm{~s}$ & 11.0 & $1.06 \mathrm{~s}$ & 16.0 & $1.04 \mathrm{~s}$ & 15.2 & $0.97 \mathrm{~s}$ \\
\hline 19 & 19.8 & $0.95 \mathrm{~s}$ & 19.8 & $0.94 \mathrm{~s}$ & 20.4 & $0.95 \mathrm{~s}$ & 12.8 & $0.70 \mathrm{~s}$ & 19.3 & $0.86 \mathrm{~s}$ \\
\hline 20 & 42.6 & $1.89 \mathrm{dq}(6.9,6.6)$ & 42.6 & $1.88 \mathrm{dq}(6.9,6.8)$ & 43.0 & $2.16 \mathrm{dq}(6.5,6.5)$ & 42.6 & $1.89 \mathrm{dq}(6.7,6.9)$ & 42.9 & $1.98 \mathrm{dq}(7.1,6.9)$ \\
\hline 21 & 13.8 & $1.32 \mathrm{~d}(6.9)$ & 13.8 & $1.31 \mathrm{~d}(6.9)$ & 14.3 & $1.41 \mathrm{~d}(6.5)$ & 13.9 & $1.32 \mathrm{~d}(6.9)$ & 13.7 & $1.38 \mathrm{~d}(6.9)$ \\
\hline 22 & 109.3 & - & 109.3 & - & 109.5 & - & 109.3 & - & 109.4 & - \\
\hline \multirow[t]{2}{*}{23} & 31.7 & $1.60 \mathrm{~m}$ & 31.7 & $1.60 \mathrm{~m}$ & 31.7 & $1.71 \mathrm{~m}$ & 31.7 & $1.60 \mathrm{~m}$ & 31.7 & $1.70(2 \mathrm{H}) \mathrm{m}$ \\
\hline & & $1.65 \mathrm{~m}$ & & $1.66 \mathrm{~m}$ & & $1.68 \mathrm{~m}$ & & $1.66 \mathrm{~m}$ & & \\
\hline 24 & 29.1 & $1.54(2 \mathrm{H}) \mathrm{m}$ & 29.1 & $1.54(2 \mathrm{H}) \mathrm{m}$ & 29.3 & $1.56(2 \mathrm{H}) \mathrm{m}$ & 29.2 & $1.53(2 \mathrm{H}) \mathrm{m}$ & 29.2 & $1.54(2 \mathrm{H}) \mathrm{m}$ \\
\hline 25 & 30.5 & $1.54 \mathrm{~m}$ & 30.5 & $1.54 \mathrm{~m}$ & 30.6 & $1.56 \mathrm{~m}$ & 30.5 & $1.54 \mathrm{~m}$ & 30.5 & $1.55 \mathrm{~m}$ \\
\hline $26_{\mathrm{ax}}$ & 66.9 & $3.45 \mathrm{dd}(10.5,11.0)$ & 66.9 & $3.45 \mathrm{dd}(10.9,10.7)$ & 66.8 & $3.52 \mathrm{dd}(10.4,10.4)$ & 66.9 & $3.46 \mathrm{dd}(11.1,9.7)$ & 66.9 & $3.46 \mathrm{dd}(11.4,9.8)$ \\
\hline $26_{\mathrm{eq}}$ & & $3.56 \mathrm{dd}(11.0,3.9)$ & & $3.56 \mathrm{dd}(10.7,3.4)$ & & $3.58 \mathrm{dd}(10.4,3.8)$ & & $3.57 \mathrm{dd}(11.1,3.7)$ & & $3.57 \mathrm{dd}(11.4,3.7)$ \\
\hline 27 & 17.3 & $0.67 \mathrm{~d}(5.9)$ & 17.2 & $0.66 \mathrm{~d}(6.0)$ & 17.3 & $0.68 \mathrm{~d}(5.6)$ & 17.3 & $0.66 \mathrm{~d}(6.0)$ & 17.3 & $0.67 \mathrm{~d}(5.9)$ \\
\hline
\end{tabular}

a Assignments were confirmed by DQF-COSY, 2D-TOCSY, HSQC, HSQC-TOCSY and HMBC experiments. 
Table 2

${ }^{13} \mathrm{C}$ and ${ }^{1} \mathrm{H}$ NMR data $\left(\mathrm{J}\right.$ in $\mathrm{Hz}$ ) of the sugar portions of compounds $\mathbf{1 - 5}$ (pyridine- $\mathrm{d}_{5}, 600 \mathrm{MHz}$ ). ${ }^{\mathrm{a}}$

\begin{tabular}{|c|c|c|c|c|c|c|c|c|c|c|}
\hline & Mague & e A (1) & Mague & e B (2) & Mague & e C (3) & Mague & He D (4) & Mague & le E (5) \\
\hline & & $\beta$-D-Gal & & $\beta$-D-Gal & & $\beta$-D-Gal & & $\beta-\mathrm{D}-\mathrm{Gal}$ & & $\beta$-D-Gal \\
\hline 1 & 103.2 & $4.89 \mathrm{~d}(7.8)$ & 103.2 & $4.89 \mathrm{~d}(7.8)$ & 103.3 & $4.89 \mathrm{~d}(7.8)$ & 103.2 & $4.88 \mathrm{~d}(7.8)$ & 103.2 & $4.89 \mathrm{~d}(7.8)$ \\
\hline 2 & 72.6 & $4.47 \mathrm{dd}(7.8,9.6)$ & 72.6 & $4.50 \mathrm{dd}(7.8,9.5)$ & 72.6 & $4.50 \mathrm{dd}(7.8,9.5)$ & 72.5 & $4.47 \mathrm{dd}(7.8,9.3)$ & 72.5 & $4.48 \mathrm{dd}(7.8,9.4)$ \\
\hline 3 & 75.3 & $4.11 \mathrm{dd}(9.6,3.0)$ & 75.4 & $4.10 \mathrm{dd}(9.5,3.2)$ & 75.5 & $4.11 \mathrm{dd}(9.5,3.9)$ & 75.4 & $4.11 \mathrm{dd}(9.3,3.3)$ & 75.4 & $4.12 \mathrm{dd}(9.4,4.0)$ \\
\hline 4 & 79.1 & $4.56 \mathrm{dd}(3.0,1.5)$ & 79.2 & $4.57 \mathrm{dd}(3.2,1.2)$ & 79.2 & $4.57 \mathrm{dd}(3.9,1.8)$ & 79.2 & $4.57 \mathrm{dd}(3.3,1.2)$ & 79.2 & $4.57 \mathrm{dd}(4.0,1.4)$ \\
\hline 5 & 75.6 & 3.99 ddd $(8.9,6.0,1.5)$ & 75.6 & 3.99 ddd $(7.6,6.3,1.2)$ & 75.6 & 3.99 ddd $(8.5,5.7,1.8)$ & 75.7 & 4.02 ddd $(8.5,6.0,1.2)$ & 75.7 & $4.02 \mathrm{ddd}(8.5,6.1,1.4)$ \\
\hline 6 & 60.5 & $\begin{array}{l}4.15 \mathrm{~m} \\
4.55 \mathrm{~m}\end{array}$ & 60.5 & $\begin{array}{l}4.15 \mathrm{~m} \\
4.57 \mathrm{~m}\end{array}$ & 60.5 & $\begin{array}{l}4.14 \mathrm{~m} \\
4.57 \mathrm{~m}\end{array}$ & 60.6 & $\begin{array}{l}4.17 \mathrm{~m} \\
4.57 \mathrm{~m}\end{array}$ & 60.6 & $\begin{array}{l}4.19 \mathrm{~m} \\
4.59 \mathrm{~m}\end{array}$ \\
\hline & & $\beta$-D-Glc & & $\beta$-D-Glc & & $\beta$-D-Glc & & $\beta$-D-Glc & & $\beta$-D-Glc \\
\hline 1 & 104.4 & $5.19 \mathrm{~d}(7.9)$ & 104.6 & $5.18 \mathrm{~d}(7.9)$ & 104.6 & $5.20 \mathrm{~d}(7.9)$ & 104.4 & $5.19 \mathrm{~d}(7.9)$ & 104.5 & $5.19 \mathrm{~d}(7.9)$ \\
\hline 2 & 80.5 & $4.31 \mathrm{dd}(7.9,8.8)$ & 81.1 & $4.33 \mathrm{dd}(7.9,8.8)$ & 81.2 & $4.33 \mathrm{dd}(7.9,8.8)$ & 80.6 & $4.32 \mathrm{dd}(7.9,8.9)$ & 80.6 & $4.33 \mathrm{dd}(7.9,8.7)$ \\
\hline 3 & 86.9 & $4.09 \mathrm{dd}(8.8,8.6)$ & 86.9 & $4.12 \mathrm{dd}(8.8,8.8)$ & 86.9 & $4.12 \mathrm{dd}(8.8,8.7)$ & 86.9 & $4.09 \mathrm{dd}(8.9,8.8)$ & 87.0 & $4.10 \mathrm{dd}(8.7,8.7)$ \\
\hline 4 & 70.3 & $3.78 \mathrm{dd}(8.6,9.3)$ & 70.3 & $3.79 \mathrm{dd}(8.8,9.3)$ & 70.4 & $3.80 \mathrm{dd}(8.7,9.2)$ & 70.3 & $3.77 \mathrm{dd}(8.8,9.5)$ & 70.3 & $3.79 \mathrm{dd}(8.7,9.5)$ \\
\hline 5 & 77.5 & $3.82 \operatorname{ddd}(9.3,7.4,2.2)$ & 77.5 & 3.82 ddd $(9.3,7.7,2.5)$ & 77.6 & 3.83 ddd $(9.2,7.3,2.2)$ & 77.5 & 3.82 ddd $(9.5,7.3,2.7)$ & 77.6 & $3.82 \mathrm{ddd}(9.5,7.5,2.6)$ \\
\hline 6 & 62.8 & $\begin{array}{l}4.04 \mathrm{dd}(10.9,7.4) \\
4.47 \mathrm{dd}(10.9,2.2)\end{array}$ & 62.8 & $\begin{array}{l}4.03 \mathrm{dd}(11.0,7.7) \\
4.48 \mathrm{dd}(11.0,2.5)\end{array}$ & 62.9 & $\begin{array}{l}4.04 \mathrm{dd}(10.3,7.3) \\
4.48 \mathrm{dd}(10.3,2.2)\end{array}$ & 62.8 & $\begin{array}{l}4.03 \mathrm{dd}(10.2,7.3) \\
4.47 \mathrm{dd}(10.2,2.7)\end{array}$ & 62.9 & $\begin{array}{l}4.04 \mathrm{dd}(10.5,7.5) \\
4.47 \mathrm{dd}(10.5,2.6)\end{array}$ \\
\hline & & $\beta-D-G l c^{\prime}$ & & $\beta$-D-Glc & & $\beta$-D-Glc & & $\beta-\mathrm{D}-\mathrm{GlC}^{\prime}$ & & $\beta$-D-Glc' \\
\hline 1 & 103.9 & $5.59 \mathrm{~d}(7.4)$ & 104.7 & $5.56 \mathrm{~d}(7.9)$ & 104.8 & $5.55 \mathrm{~d}(7.9)$ & 103.9 & $5.60 \mathrm{~d}(7.5)$ & 103.9 & $5.61 \mathrm{~d}(7.6)$ \\
\hline 2 & 75.0 & $4.04 \mathrm{dd}(7.4,9.2)$ & 76.0 & $4.02 \mathrm{dd}(7.9,9.5)$ & 76.0 & $4.03 \mathrm{dd}(7.9,9.3)$ & 75.0 & $4.04 \mathrm{dd}(7.5,9.9)$ & 75.0 & $4.04 \mathrm{dd}(7.6,9.0)$ \\
\hline 3 & 87.0 & $4.07 \mathrm{dd}(9.2,8.9)$ & 78.0 & $4.12 \mathrm{dd}(9.5,9.3)$ & 78.1 & $4.12 \mathrm{dd}(9.3,8.2)$ & 87.0 & $4.08 \mathrm{dd}(9.9,9.0)$ & 87.0 & $4.08 \mathrm{dd}(9.0,9.0)$ \\
\hline 4 & 69.3 & $3.93 \mathrm{dd}(8.9,9.3)$ & 71.3 & $4.07 \mathrm{dd}(9.3,8.7)$ & 71.3 & $4.07 \mathrm{dd}(8.2,9.3)$ & 69.4 & $3.92 \mathrm{dd}(9.0,8.8)$ & 69.4 & $3.93 \mathrm{dd}(9.0,9.1)$ \\
\hline 5 & 77.9 & $3.85 \mathrm{ddd}(9.3,6.0,2.4)$ & 78.4 & 3.89 ddd $(8.7,5.5,2.3)$ & 78.4 & 3.88 ddd $(9.3,5.5,2.2)$ & 77.9 & 3.85 ddd $(8.8,5.7,1.9)$ & 77.9 & $3.85 \mathrm{ddd}(9.1,5.0,2.5)$ \\
\hline 6 & 62.3 & $\begin{array}{l}4.29 \mathrm{dd}(11.7,5.0) \\
4.46 \mathrm{dd}(11.7,3.1)\end{array}$ & 62.6 & $\begin{array}{l}4.38 \mathrm{dd}(12.0,5.5) \\
4.54 \mathrm{dd}(12.0,2.3)\end{array}$ & 62.7 & $\begin{array}{l}4.37 \mathrm{dd}(12.5,5.5) \\
4.53 \mathrm{dd}(12.5,2.2)\end{array}$ & 62.3 & $\begin{array}{l}4.30 \mathrm{dd}(12.0,5.7) \\
4.47 \mathrm{dd}(12.0,1.9)\end{array}$ & 62.3 & $\begin{array}{l}4.30 \mathrm{dd}(11.9,5.0) \\
4.47 \mathrm{dd}(11.9,2.5)\end{array}$ \\
\hline & & $\beta-D-X y l$ & & $\beta-\mathrm{D}-\mathrm{Xyl}$ & & $\beta-\mathrm{D}-\mathrm{Xyl}$ & & $\beta-\mathrm{D}-\mathrm{Xyl}$ & & $\beta-\mathrm{D}-\mathrm{Xyl}$ \\
\hline 1 & 104.8 & $5.15 \mathrm{~d}(7.8)$ & 104.9 & $5.22 \mathrm{~d}(7.8)$ & 104.9 & $5.23 \mathrm{~d}(7.8)$ & 104.8 & $5.15 \mathrm{~d}(7.8)$ & 104.8 & $5.16 \mathrm{~d}(7.7)$ \\
\hline 2 & 75.1 & $3.93 \mathrm{dd}(7.8,8.5)$ & 75.1 & $3.93 \mathrm{dd}(7.8,8.4)$ & 75.1 & $3.93 \mathrm{dd}(7.8,8.4)$ & 75.1 & $3.93 \mathrm{dd}(7.8,8.6)$ & 75.1 & $3.93 \mathrm{dd}(7.7,8.3)$ \\
\hline 3 & 78.4 & $4.04 \mathrm{dd}(8.5,8.9)$ & 78.6 & $4.06 \mathrm{dd}(8.4,8.9)$ & 78.7 & $4.06 \mathrm{dd}(8.4,8.8)$ & 78.4 & $4.04 \mathrm{dd}(8.6,8.9)$ & 78.4 & $4.04 \mathrm{dd}(8.3,9.0)$ \\
\hline 4 & 70.7 & $4.08 \mathrm{~m}$ & 70.7 & $4.10 \mathrm{~m}$ & 70.7 & $4.09 \mathrm{~m}$ & 70.7 & $4.08 \mathrm{~m}$ & 70.7 & $4.07 \mathrm{~m}$ \\
\hline $5_{\mathrm{ax}}$ & 67.2 & $3.62 \mathrm{dd}(11.2,10.4)$ & 67.3 & $3.64 \mathrm{dd}(11.2,10.2)$ & 67.3 & $3.64 \mathrm{dd}(11.2,10.3)$ & 67.2 & $3.61 \mathrm{dd}(11.4,9.9)$ & 67.2 & $3.62 \mathrm{t}(11.2)$ \\
\hline $5_{\mathrm{eq}}$ & & $4.18 \mathrm{dd}(11.2,4.9)$ & & $4.19 \mathrm{dd}(11.2,4.9)$ & & $4.20 \mathrm{dd}(11.2,4.9)$ & & $4.18 \mathrm{dd}(11.4,4.9)$ & & $4.18 \mathrm{dd}(11.2,4.9)$ \\
\hline & & $\beta-\mathrm{D}-\mathrm{Xyl}^{\prime}$ & & & & & & $\beta-\mathrm{D}-\mathrm{Xyl}^{\prime}$ & & $\beta-\mathrm{D}-\mathrm{Xyl}^{\prime}$ \\
\hline 1 & 106.1 & $5.06 \mathrm{~d}(7.4)$ & & & & & 106.1 & $5.06 \mathrm{~d}(7.4)$ & 106.1 & $5.06 \mathrm{~d}(7.4)$ \\
\hline 2 & 75.3 & $3.91 \mathrm{dd}(7.4,8.6)$ & & & & & 75.4 & $3.91 \mathrm{dd}(7.4,8.5)$ & 75.4 & $3.91 \mathrm{dd}(7.4,8.1)$ \\
\hline 3 & 77.7 & $4.01 \mathrm{dd}(8.6,8.6)$ & & & & & 77.7 & $4.01 \mathrm{dd}(8.5,8.6)$ & 77.7 & $4.01 \mathrm{dd}(8.1,8.6)$ \\
\hline 4 & 70.7 & $4.08 \mathrm{~m}$ & & & & & 70.7 & $4.08 \mathrm{~m}$ & 70.7 & $4.08 \mathrm{~m}$ \\
\hline $5_{\mathrm{ax}}$ & 67.0 & $3.46 \mathrm{dd}(11.1,10.6)$ & & & & & 67.0 & $3.46 \mathrm{dd}(11.6,9.9)$ & 67.1 & $3.46 \mathrm{t}(11.4)$ \\
\hline $5_{\text {eq }}$ & & $4.16 \mathrm{dd}(11.1,5.3)$ & & & & & & $4.16 \mathrm{dd}(11.6,5.2)$ & & $4.16 \mathrm{dd}(11.4,5.1)$ \\
\hline
\end{tabular}

a Assignments were confirmed by DQF-COSY, 1D-TOCSY, HSQC, HSQC-TOCSY and HMBC experiments. 


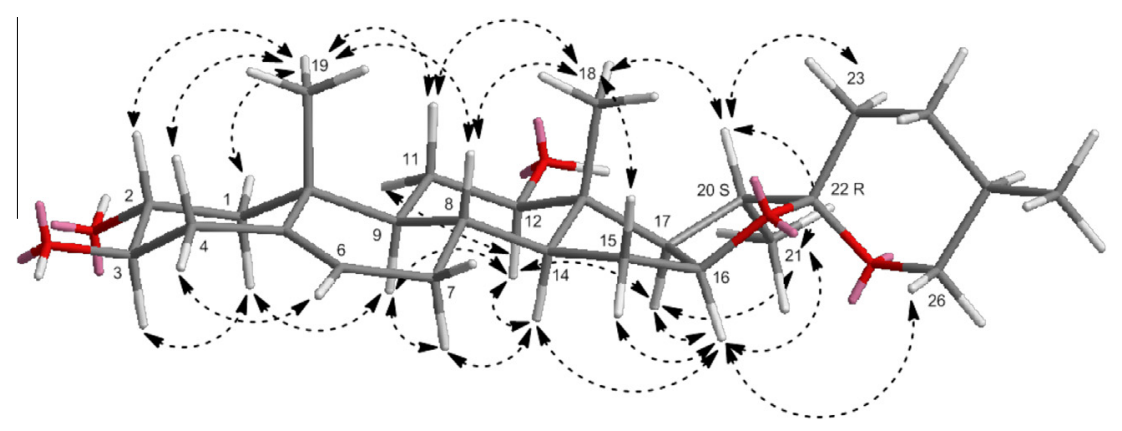

Fig. 4. Main correlations observed in 2D-ROESY spectrum for aglycone moiety of 3.

The fractions bioassay (Fig. 2) showed a roots growth inhibition as general behavior, being L. sativa the most affected species. Based on the available quantities of the saponins, lettuce was chosen as a model to know their activities, testing at 333; 100; 33; 10; 3.3 and $1 \mu \mathrm{M}$. Magueyoside C (3) and compounds $\mathbf{1 0}$ and $\mathbf{1 1}$ could not be assayed.

The results of the bioassay are shown in Fig. 5, where data are presented as percentage from the control. Positive values indicate stimulation and negative values represent an inhibition of the studied parameters. Due to the fact that effects on germination and shoot development were not significant, the discussion was performed on the base of roots growth effects.

Inhibitory effects at concentrations higher than $100 \mu \mathrm{M}$ and stimulatory effects at concentration lower than $33 \mu \mathrm{M}$ of tested compounds were observed. Compounds 1, 2 and 4-7 presented better inhibition profiling than shown by the commercial herbicide LOGRAN $^{\circledR}$. YS-IX (6) showed the lowest value of $\mathrm{IC}_{50} 66.7 \mu \mathrm{M}$ (Table 3 ). In addition, the growth inhibition is more prominent in $\mathbf{1 , 2}$ and 4-6, showing an average $\mathrm{IC}_{50}$ of $100 \mu \mathrm{M}$, which could be possible correlate with the presence of the carbonyl group at C-12 of their aglycones.

On the other hand, it is not completely possible to find a correlation between 12-keto-saponins bioactivity and units number in sugar moiety, nonetheless the comparison of the profiling of $\mathbf{7}$ and 8 allowed to observe a better inhibition profile ( $\mathrm{IC}_{50}$ $160.2 \mu \mathrm{M}$ ) for this with five sugar units, Agabrittonoside A (7), than that with four sugar units, Karatavioside $A(8)\left(\mathrm{IC}_{50} 700.1 \mu \mathrm{M}\right)$.

Likewise, stimulatory effects at low concentrations were observed. Saponins $\mathbf{1}$ and $\mathbf{9}$ showed the best profiles with maximum of $16 \%$ and $19 \%(P<0.01)$ of stimulatory response at $10 \mu \mathrm{M}$.

Biphasic dose-response relationships, which are characterized by a stimulatory response in the measured parameter at low doses of active compound and inhibition at higher doses, have been recognized in plants, whether effects of synthetic herbicides or

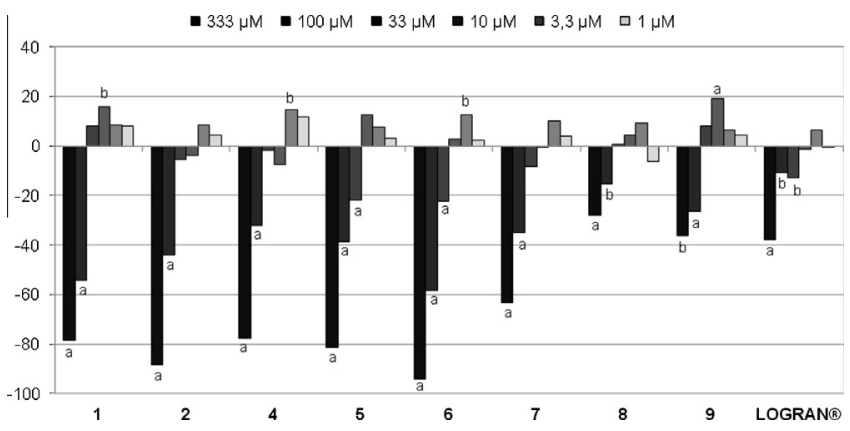

Fig. 5. Effects of compounds 1, 2 and 4-9 on roots growth of Lactuca sativa L. Values are expressed as percentage from the control and are not significantly different with

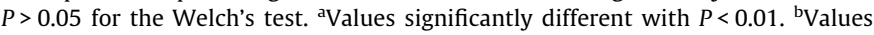
significantly different with $0.01<P<0.05$.
Table 3

Phytotoxicity of compounds 1, 2 and 4-9 on roots of Lactuca sativa L.

\begin{tabular}{lll}
\hline Compounds & $\mathrm{IC}_{50}(\mu \mathrm{M})$ & $r^{2}$ \\
\hline $\mathbf{1}$ & 88.4 & 0.9689 \\
$\mathbf{2}$ & 104.3 & 0.9714 \\
$\mathbf{4}$ & 131.2 & 0.9706 \\
$\mathbf{5}$ & 101.6 & 0.9729 \\
$\mathbf{6}$ & 66.7 & 0.9802 \\
$\mathbf{7}$ & 160.2 & 0.9964 \\
$\mathbf{8}$ & $700.1^{*}$ & 0.9806 \\
$\mathbf{9}$ & $375.9^{*}$ & 0.9510 \\
LOGRAN $^{\circledR}$ & $523.7^{*}$ & 0.9863 \\
\hline
\end{tabular}

The data were not adjusted to the dose-response curve.

natural phytotoxins are concerned (Duke et al., 2006). This doseresponse phenomenon is known as hormesis, which has been described for oleanane saponins, in special for those isolated from mungbeans (Waller et al., 1996).

In conclusion, we can state that $A$. offoyana is an interesting source of active compounds. The 12-ketosaponins presented the highest levels of roots growth inhibition better than shown by the commercial herbicide Logran ${ }^{\infty}$. On the other hand, low concentrations of saponins enhance the root growth.

\section{Experimental}

\subsection{General experimental procedure}

Optical rotations were carried out using a Perkin-Elmer 241 polarimeter $\left(589 \mathrm{~nm}, 20^{\circ} \mathrm{C}\right)$. 1D and 2D NMR spectra were recorded on a Varian INOVA-600 spectrometer equipped with $5 \mathrm{~mm}{ }^{1} \mathrm{H}\left\{{ }^{15} \mathrm{~N}-{ }^{31} \mathrm{P}\right\}$ PFG high-field inverse detection z-gradient probe. ${ }^{1} \mathrm{H}(599.78 \mathrm{MHz})$ and ${ }^{13} \mathrm{C}(150.83 \mathrm{MHz})$ NMR spectra were recorded in pyridine- $d_{5}$ at $25{ }^{\circ} \mathrm{C}$. Chemical shift are given on the $\delta$ scale and were referenced to residual pyridine, $\delta_{\mathrm{H}} 8.70,7.55$, 7.18 and $\delta_{\mathrm{C}} 149.84,135.50,123.48$. Varian pulse sequence using gradient were applied and all 2D spectra, except for HMBC, were recorded in the phase-sensitive mode. HR-FAB-MS data were detected on a Waters AUTOSPEC mass spectrometer. HPLC in isocratic mode was performed by a Merck Hitachi apparatus equipped with LaChrom (L-2490) refractive index detector and analytical Phenomenex Gemini C18 column $(4.6 \times 250 \mathrm{~mm}$, i.d).

\subsection{Plant material}

Flowers of A. offoyana were collected on January 2008 by botanist Dr. Alfredo Noa in Palenque, Remedios city, north of Villa Clara province, Cuba. A voucher specimen was deposited in the Herbarium Dr. Alberto Alonso Triana of the Universidad Central 'Marta Abreu' de Las Villas, Cuba (number HPVC 3017). 


\subsection{Extraction and isolation}

Dried and powdered flowers $(0.5 \mathrm{~kg})$ were extracted three times with ethanol-water ( $7: 3$ ) during $48 \mathrm{~h}$ by maceration at room temperature. The solvent was removed under reduced pressure and the syrupy extract (11.4\%) was suspended in distilled water, defatted with $n$-hexane, and then extracted with water-saturated $n$ $\mathrm{BuOH}$. After removing the solvent, $10 \mathrm{~g}$ of $n-\mathrm{BuOH}$ extract $(30.8 \%$, of ethanolic extract) was purified by VLC over LiChrospher RP-18 and eluted with a $\mathrm{MeOH}-\mathrm{H}_{2} \mathrm{O}(4: 6,5: 5,6: 4,7: 3,8: 2,10: 0$, each $250 \mathrm{ml}$ ), to give five fractions (Fr A: $2.13 \mathrm{~g}, \mathrm{Fr} \mathrm{B}: 0.87 \mathrm{~g}$, Fr C: $0.29 \mathrm{~g}$, Fr D: $0.69 \mathrm{~g}$, and Fr E: $6.0 \mathrm{~g}$ ).

Fr D $(0.69 \mathrm{~g})$ was subjected to MPLC $(1 \mathrm{ml} / \mathrm{min})$ on a Büchi 861 apparatus onto, 40-63 $\mu \mathrm{m}$ LiChrospher RP-18 column, using Acetone- $\mathrm{H}_{2} \mathrm{O}(1: 1)$ as mobile phase. Six milliliter fractions were collected and checked by TLC on RP-18 $\mathrm{F}_{254 \mathrm{~S}}$, developed with $\mathrm{MeOH}-\mathrm{H}_{2} \mathrm{O}(8: 2)$ and sprayed with Oleum reagent and heated at $150{ }^{\circ} \mathrm{C}$. Fractions showing similar profiling were combined to give 6 fractions, from Fr D-3 to Fr D-5, each comprising two to four saponins.

Fractionation of Fr D-3 by HPLC on analytical C18 column and $\mathrm{MeOH}-\mathrm{H}_{2} \mathrm{O}$ (7:3) as mobile phase yielded two further fractions, which were purified by HPLC using $\mathrm{ACN}-\mathrm{H}_{2} \mathrm{O}$ (3.5:6.5) and the same column to afford compounds $\mathbf{3}(2.7 \mathrm{mg})$ and $\mathbf{5}(3.3 \mathrm{mg})$.

In the same conditions described above Fr D-4 was fractionated by HPLC to obtain 3 additional fractions. The first one gave compound $1(9.4 \mathrm{mg})$ after further purification by means of HPLC analytical $\mathrm{C} 18$ column and $\mathrm{ACN}-\mathrm{H}_{2} \mathrm{O}$ (3.5:6.5) as mobile phase. Using the same HPLC column but $\mathrm{MeOH}-\mathrm{H}_{2} \mathrm{O}(7: 3)$ as mobile phase allowed obtain compound $6(3.9 \mathrm{mg})$ from the third fraction and a preparative TLC silica gel EtOAc-HOAc- $\mathrm{H}_{2} \mathrm{O}(7: 2: 2)$, for the second one gave compounds 2 ( $4.1 \mathrm{mg})$ and $4(3.5 \mathrm{mg})$.

From Fr D-5, the compounds $\mathbf{9}(4.5 \mathrm{mg}), \mathbf{1 0}(1.2 \mathrm{mg})$ and $\mathbf{1 1}$ $(1.4 \mathrm{mg})$ were purified by HPLC, analytical C18 column and $\mathrm{MeOH}-\mathrm{H}_{2} \mathrm{O}(8: 2)$ as mobile phase.

Fr E (6.0 g) was chromatographed by $\mathrm{CC}$ silica gel $\mathrm{CHCl}_{3}-$ $\mathrm{MeOH}-\mathrm{H}_{2} \mathrm{O}$ (30:10:1 to 7:4:1) and purified by HPLC on analytical C18 column ( $1 \mathrm{ml} / \mathrm{min})$ with $\mathrm{MeOH}-\mathrm{H}_{2} \mathrm{O}(7: 3)$ as mobile phase to afford compounds 7 (10.5 $\mathrm{mg}$ ) and 8 ( $8 \mathrm{mg}$ ).

\subsection{Compound 1: $[\alpha]_{D}^{20}-43.5(\mathrm{MeOH}$, c 0.1$)$}

HRFABMS, $m / z$ 1217.5201 [M+Na] ${ }^{+}$calculated for $\mathrm{C}_{55} \mathrm{H}_{86} \mathrm{O}_{28} \mathrm{Na}$, 1217.5203. ${ }^{1} \mathrm{H}$ and ${ }^{13} \mathrm{C}$ NMR reported in Tables 1 and 2.

3.5. Compound 2: $[\alpha]_{D}^{20}-33.5(\mathrm{MeOH}, \mathrm{c} 0.1)$

HRFABMS, $m / z$ 1085.4830 [M+Na] $]^{+}$calculated for $\mathrm{C}_{50} \mathrm{H}_{78} \mathrm{O}_{24} \mathrm{Na}$, 1085.4781. ${ }^{1} \mathrm{H}$ and ${ }^{13} \mathrm{C}$ NMR reported in Tables 1 and 2.

3.6. Compound 3: $[\alpha]_{D}^{20}-79.2(\mathrm{MeOH}$, c 0.1$)$

HRFABMS, $m / z$ 1087.4919 [M+Na] $]^{+}$calculated for $\mathrm{C}_{50} \mathrm{H}_{80} \mathrm{O}_{24} \mathrm{Na}$, 1087.4937. ${ }^{1} \mathrm{H}$ and ${ }^{13} \mathrm{C}$ NMR reported in Tables 1 and 2.

\subsection{Compound 4: $[\alpha]_{D}^{20}-18,2(\mathrm{MeOH}, \mathrm{c} 0.1)$}

HRFABMS, $m / z 1219.5406[\mathrm{M}+\mathrm{Na}]^{+}$calculated for $\mathrm{C}_{55} \mathrm{H}_{88} \mathrm{O}_{28} \mathrm{Na}$, 1219.5360. ${ }^{1} \mathrm{H}$ and ${ }^{13} \mathrm{C}$ NMR reported in Tables 1 and 2 .

\subsection{Compound 5: $[\alpha]_{D}^{20}-44.7(\mathrm{MeOH}$, c 0.1$)$}

HRFABMS, $m / z 1217.5211[\mathrm{M}+\mathrm{Na}]^{+}$calculated for $\mathrm{C}_{55} \mathrm{H}_{86} \mathrm{O}_{28} \mathrm{Na}$, 1217.5203. ${ }^{1} \mathrm{H}$ and ${ }^{13} \mathrm{C}$ NMR reported in Tables 1 and 2 .
3.9. Compound 7: $[\alpha]_{D}^{20}-50.5(\mathrm{MeOH}$, c 0.1$)$

HRFABMS, $m / z 1203.5411[\mathrm{M}+\mathrm{Na}]^{+}$calculated for $\mathrm{C}_{55} \mathrm{H}_{88} \mathrm{O}_{27} \mathrm{Na}$, 1203.5411.

\subsection{Acid Hydrolysis of saponins}

Compounds 1-5 and 7 (each $2 \mathrm{mg}$ ) were treated with $1 \mathrm{~N} \mathrm{HCl}$ $(1 \mathrm{ml})$ at $80^{\circ} \mathrm{C}$ for $3 \mathrm{~h}$. After cooling, the solvent was eliminated with a stream of $\mathrm{N}_{2}$, dry residue was suspended in water, and aglycones were extracted with ethyl acetate $(3 \times 2 \mathrm{ml})$. The aqueous layer, containing sugars, was neutralized with Amberlite IRA-400 $\left(\mathrm{OH}^{-}\right.$form $)$. For each samples, aqueous solution were dried under $\mathrm{N}_{2}$ and stored for the subsequent analysis.

\subsection{Determination of absolute configurations of sugars}

To determine the absolute configuration of monosaccharide constituents of isolated compounds 1-5 and 7, the method reported by (Tanaka et al., 2007) was used with slight modifications. Sugars of each sample were dissolved in pyridine $(0.5 \mathrm{~mL})$ containing L-cysteine methyl ester hydrochloride $(2 \mathrm{mg})$ and heated at $60^{\circ} \mathrm{C}$ for $1 \mathrm{~h}$; o-tolyl isothiocyanate $(2 \mu \mathrm{l})$ was then added and the mixture was heated at $60^{\circ} \mathrm{C}$ for $1 \mathrm{~h}$. Each reaction mixture was directly analyzed by reversed-phase HPLC using a model 616 pump, a 996 photodiode array detector and a 717-plus autosampler (Waters, Milford, MA). An Eurospher-100 $C_{18}$, $250 \mathrm{~mm} \times 4 \mathrm{~mm}$ i.d., $5 \mu \mathrm{m}$ (Knauer, Berlin, Germany) HPLC column was used; mobile phase: $\mathrm{MeCN}-\mathrm{H}_{2} \mathrm{O}(25: 75, \mathrm{v} / \mathrm{v})$ containing $50 \mathrm{mM} \mathrm{H}_{3} \mathrm{PO}_{4}$; detection: $\mathrm{UV}(250 \mathrm{~nm})$; flow rate: $0.8 \mathrm{ml} / \mathrm{min}$; column temperature: $35^{\circ} \mathrm{C}$. The HPLC column was washed with $\mathrm{MeOH}$ after each injection.

The derivatives of monosaccharides of D-xylose, D-glucose and $\mathrm{D}$-galactose, in the analyzed saponins were identified by comparison of their retention times with those of authentic samples (Sigma-Aldrich, Steinheim, Germany) treated in the same way as described above (Rt: D-xylose $17.69 \mathrm{~min}$, L-xylose $16.27 \mathrm{~min}$, D-glucose $14.57 \mathrm{~min}$, L-glucose $13.43 \mathrm{~min}$, D-galactose $12.63 \mathrm{~min}$, L-galactose $13.13 \mathrm{~min})$.

\subsection{Coleoptiles bioassay}

Wheat seeds (T. aestivum L. cv. Duro) were sown in $15 \mathrm{~cm}$ diameter Petri dishes moistened with water and grown in the dark at $22 \pm 1{ }^{\circ} \mathrm{C}$ for 3 days (Hancock et al., 1964). The roots and caryopsis were removed from the shoots. Then the shoots were placed in a Van der Weij guillotine and the apical $2 \mathrm{~mm}$ were cut off and discarded.

The next $4 \mathrm{~mm}$ of the coleoptiles were removed and used for the bioassay. All manipulations were performed under a green safelight (Nitsch and Nitsch, 1956). Products of interest were previously dissolved in DMSO and diluted to the final bioassay concentration with a maximum of $0.5 \%$ DMSO. Parallel controls were also run.

Crude extracts and fractions to be assayed for biological activity were added to test tubes. The assays were carried out in duplicate. Phosphate-citrate buffer $(2 \mathrm{ml})$ containing $2 \%$ sucrose (Nitsch and Nitsch, 1956) at pH 5.6 was added to each test tube. Five coleoptiles were placed in each test tube (three tubes per dilution) and the tubes were rotated at $6 \mathrm{rpm}$ in a roller tube apparatus for $24 \mathrm{~h}$ at $22^{\circ} \mathrm{C}$ in the dark. The coleoptiles were measured by digitalization of their images. Data were statistically analyzed using Welch's test (Martín Andrés and Luna del Castillo, 1990). Data are presented as percentage differences from control, where zero represents the control, positive values represent stimulation of the studied parameter, and negative values represent inhibition. 


\subsection{Phytotoxicity bioassay}

Selection of target plants was based on an optimization process developed by us in our search for a standard phytotoxicity bioassay (Macías et al., 2000). Several Standard Target Species (STS) were proposed, including monocot $A$. cepa L. (onion) and dicots $L$. esculentum Will. (tomato), L. sativum L. (cress) and L. sativa L. (lettuce), which were assayed for this study.

Bioassays were conducted using Petri dishes (50 mm diameter), with one sheet of Whatman No.1 filter paper as support. Germination and growth were conducted in aqueous solutions at controlled $\mathrm{pH}$ using $10^{-2} \mathrm{M}$ 2-[N-morpholino]ethanesulfonic acid (MES) and $1 \mathrm{M} \mathrm{NaOH}$ ( $\mathrm{pH}$ 5.6). Fractions and compounds to be assayed were dissolved in DMSO and these solutions were diluted with buffer (with a maximum of $0.5 \%$ DMSO), so that test concentrations for fractions (800, 400 and $200 \mathrm{ppm}$ ) and each compound (333, 100, $33,10,3,3,1 \mu \mathrm{M})$ were reached. This procedure facilitated the solubility of the assayed compounds. 20 seeds were used to each Petri dish and four replicates were made ( 80 seeds in total). Treatment, control or internal reference solution $(1 \mathrm{ml})$ was added to each Petri dish.

After adding seeds and aqueous solutions, Petri dishes were sealed with Parafilm to ensure closed-system models. Seeds were further incubated at $25^{\circ} \mathrm{C}$ in a Memmert ICE 700 controlled environment growth chamber in the dark. Bioassays took 4 days for cress, 5 days for lettuce and tomato, and 7 days for onion. After growth, plants were frozen at $-10{ }^{\circ} \mathrm{C}$ for 24 h to avoid subsequent growth during the measurement process.

The commercial herbicide $\operatorname{Logran}^{\circledR}$, a combination of $\mathrm{N}-(1,1-$ dimethylethyl)- $N^{\prime}$-ethyl-6-(methylthio)-1,3,5-triazine-2,4-diamine (Terbutryn, 59.4\%) and 2-(2-chloroethoxy)-N-\{[(4-methoxy-6methyl-1,3,5-triazin-2yl)amino]carbonyl \}benzene-sulfonamide (Triasulfuron, $0.6 \%$ ), was used as an internal reference according the comparison study reported previously (Macías et al., 2000). This was tested in the same way as described above for the samples.

Control samples (buffered aqueous solutions with DMSO and without any test compound) were used for all of the plant species assayed.

Evaluated parameters (germination rate, root length and shoot length) were recorded using a Fitomed ${ }^{\circledR}$ system (Castellano et al., 2001), which allowed automatic data acquisition and statistical analysis using its associated software. Data were analyzed statistically using Welch's test, with significance fixed at 0.01 and 0.05 .

Results are presented as percentage differences from the control. Zero represents control, positive values represent stimulation, and negative values represent inhibition. $\mathrm{IC}_{50}$ values to inhibition effects were obtained after adjusting phytotoxicity data to concentration (logarithmic scale), to a sigmoidal dose-response curve, defined by equation:

$Y=Y_{\min }+\frac{Y_{\min }-Y_{\min }}{1+10^{\log g E C 0-x}}$

where $X$ indicates the logarithm of concentration, $Y$ indicates the response (phytotoxicity) and $Y_{\max }$ and $Y_{\min }$ are the maximum and minimum values of the response, respectively. Goodness of fit is described by the determination coefficient $\left(r^{2}\right)$. The adjustment and the $r^{2}$ were obtained using GraphPad Prism $5.0^{\circledR}$ software.

\section{Acknowledgements}

This research was supported by the Ministerio de Educación y Ciencia (Project No. AGL2009-08864 (AGR)) and Junta de Andalucía (PAI-III, AGR-5822), Seville, Spain. A fellowship from the Agencia
Española de Cooperación Internacional para el Desarrollo, AECID (AJP) is also gratefully acknowledged.

\section{Appendix A. Supplementary data}

Supplementary data associated with this article include NMR spectra $\left({ }^{1} \mathrm{H}\right.$ and ${ }^{13} \mathrm{C}$ NMR, HSQC, HMBC, ROESY and DQF-COSY) for the new compounds (1-5), tables with complete spectroscopy data of known compounds 6, 9-11 which have not been reported so far, graphics of the effects of compounds 1, 2 and 4-9 on germination and shoots growth of Lactuca sativa and a table with the phytotoxicity values of these compounds. Supplementary data associated with this article can be found, in the online version, at http://dx.doi.org/10.1016/j.phytochem.2013.06.020.

\section{References}

Abdel Gawad, M.M., El Sayed, M.M., Abdel Hameed, E.S., 1999. Molluscicidal steroidal saponins and lipid content of Agave decipiens. Fitoterapia 70, 371-381. Álvarez de Zayas, A., 1996. Los agaves de Cuba central. Fontqueria 44, 117-128.

Blunden, G., Yi, Y., Jewers, K., Burbage, M.B., 1974. Agave ghiesbrechtii, a new source of gloriogenin. Phytochemistry 13, 2025-2027.

Blunden, G., Patel, A.V., Crabb, T.A., 1986. Barbourgenin, a new steroidal sapogenin from Agave sisalana leaves. J. Nat. Prod. 49, 687-689.

Castellano, D., Macías, F.A., Castellano, M., Cambronero, R., 2001. Sistema automatizado para la adquisición simultánea y gestión informatizada de medidas de longitud variable. Spanish Patent No. P9901565.

Chen, P.Y., Chen, C.H., Kuo, C.C., Lee, T.H., Kuo, Y.H., Lee, C.K., 2011. Cytotoxic steroidal saponins from Agave sisalana. Planta Med. 77, 929-933.

Chen, P.Y., Kuo, Y.C., Chen, C.H., Kuo, Y.H., Lee, C.K., 2009. Isolation and immunomodulatory effect of homoisoflavones and flavones from Agave sisalana Perrine ex Engelm. Molecules 14, 1789-1795.

Ding, Y., Chen, Y.Y., Wang, D.Z., Yang, C.R., 1989. Steroidal saponins from a cultivated form of Agave sisalana. Phytochemistry 28, 2787-2791.

Ding, Y., Tian, R.H., Yang, C.R., Chen, Y.Y., Nohara, T., 1993. Two new steroidal saponins from dried fermented residues of leaf-juices of Agave sisalana forma Dong No. 1. Chem. Pharm. Bull. 41, 557-560.

Duke, S.O., Cedergreen, N., Velini, E.D., Belz, R.G., 2006. Hormesis: is it an important factor in herbicide use and allelopathy? Outlooks Pest Manag. 17, 29-33.

Eskander, J., Lavaud, C., Harakat, D., 2010. Steroidal saponins from the leaves of Agave macroacantha. Fitoterapia 81, 371-374.

Hancock, C.R., Barlow, H.W., Lacey, H.J., 1964. The east malling coleoptile straight growth test method. J. Exp. Bot. 15, 166-176.

Helmkamp, G.K., Bornner, J., 1953. Some relationships of sterols to plant growth. Plant Physiol. 28, 428-435.

Hernández Carlos, B., González Coloma, A., Orozco Valencia, A.U., Ramírez Mares, M.V., Andrés Yeves, M.F., Joseph Nathan, P., 2011. Bioactive saponins from Microsechium helleri and Sicyos bulbosus. Phytochemistry 72, 743-751.

Itabashi, M., Segawa, K., Ikeda, Y., Kondo, S., Naganawa, H., Koyano, T., Umezawa, K., 2000. A new bioactive steroidal saponin, furcreastatin, from the plant Furcraea foetida. Carbohydr. Res. 323, 57-62.

Jain, D.C., 1987. Gintogenin-3-O- $\beta$-D-laminaribioside from the aerial part of Agave cantala. Phytochemistry 26, 1789-1790.

Jin, J.M., Liu, X.K., Teng, R.W., Yang, C.R., 2002a. Two new steroidal glycosides from fermented leaves of Agave americana. Chin. Chem. Lett. 13, 629-632.

Jin, J.M., Liu, X.K., Yang, C.R., 2002b. A new pregnane glycoside from fermented leaves of Agave americana. Chin. Chem. Lett. 13, 1189-1192.

Jin, J.M., Yang, C.R., 2003a. Two new spirostanol steroidal sapogenins from fermented leaves of Agave americana. Chin. Chem. Lett. 14, 491-494.

Jin, J.M., Liu, X.K., Yang, C.R., 2003b. Three new hecogenin glycosides from fermented leaves of Agave americana. J. Asian Nat. Prod. Res. 5, 95-103.

Jin, J.M., Zhang, Y.J., Yang, C.R., 2004. Four new steroid constituents from the waste residue of fibre separation from Agave americana leaves. Chem. Pharm. Bull. 52, 654-658.

Kang, L.P., Zhang, J., Cong, Y., Li, B., Xiong, C.Q., Zhao, Y., Tan, D.W., Yu, H.S., Yu, Z.Y., Cong, Y.W., Liu, C., Ma, B.P., 2012. Steroidal glycosides from the rhizomes of Anemarrhena asphodeloides and their antiplatelet aggregation activity. Planta Med. 78, 611-616.

Killeen, G.F., Madigan, C.A., Connolly, C.R., Walsh, G.A., Clark, C., Hynes, M.J., Timmins, B.F., James, P., Headon, D.R., Power, R.F., 1998. Antimicrobial saponins of Yucca schidigera and the implications of their in vitro properties for their in vivo impact. J. Agric. Food Chem. 46, 3179-3186.

Li, H., Huang, W., Wen, Y., Gong, G., Zhao, Q., Yu, G., 2010. Anti-thrombotic activity and chemical characterization of steroidal saponins from Dioscorea zingiberensis C.H. Wright. Fitoterapia 8, 1147-1156.

Macías, F.A., Castellano, D., Molinillo, J.M.G., 2000. Search for a standard phytotoxic bioassay for allelochemicals. Selection of standard target species. J. Agric. Food Chem. 48, 2512-2521.

Macías, F.A., Guerra, J.O., Simonet, A.M., Nogueiras, C.M., 2007. Characterization of the fraction components using 1D TOCSY and 1D ROESY experiments. Four new 
spirostane saponins from Agave brittoniana Trel. spp. Brachypus. Magn. Reson. Chem. 45, 615-620.

Macías, F.A., Guerra, J.O., Simonet, A.M., Pérez, A.J., Nogueiras, C.M., 2010. Characterization of three saponins from a fraction using 1D DOSY as a solvent signal suppression tool. Agabrittonosides E-F. Furostane Saponins from Agave brittoniana Trel. spp. Brachypus. Magn. Reson. Chem. 48, 350-355.

Marker, R.E., Wagner, R.B., Ulshafer, P.R., Wittbecker, E.L., Goldsmith, D.P.J., Ruof, C.H., 1943. Sterols. CLVII. Sapogenins. LXM'. Isolation and structures of thirteen new steroidal sapogenins. New sources for known sapogenins. J. Am. Chem. Soc. 65, 1199-1209.

Martín Andrés, A., Luna el Castillo, J.De.D., 1990. Bioestadística Para Las Ciencias de la Salud, third ed. Norma-Capitel, Madrid.

Mimaki, Y., Kanmoto, T., Kuroda, M., Sashida, Y., Nishino, A., Satomi, Y., Nishino, H., 1995. Steroidal saponins from the underground parts of Hosta longipes and their inhibitory activity on tumor promoter-induced phospholipid metabolism. Chem. Pharm. Bull. 43, 1190-1196.

Mimaki, Y., Kuroda, M., Kameyama, A., Yokosuka, A., Sashida, Y., 1998. Steroidal saponins from the rhizomes of Hosta sieboldii and their citostatic activity on HL60 cells. Phytochemistry 48, 1361-1369.

Mimaki, Y., Yokosuka, A., Sashida, Y., 2000. Steroidal glycosides from the aerial parts of Polianthes tuberosa. J. Nat. Prod. 63, 1519-1523.

Morales Serna, J.A., Jiménez, A., Estrada Reyes, R., Márquez, C., Cárdenas, J., Salmón, M., 2010. Homoisoflavanones from Agave tequilana Weber. Molecules 15, 32953301.

Nakano, K., Midzuta, Y., Hara, Y., Murakami, K., Takaishi, Y., Tomimatsu, T., 1991. 12Keto steroidal glycosides from the caudex of Yucca gloriosa. Phytochemistry 30, 633-636.

Nitsch, J.P., Nitsch, C., 1956. Studies on the growth of coleoptile and first internode sections. A new sensitive, straight-growth test for auxins. Plant Physiol. 31, 94111.

Ohtsuki, T., Koyano, T., Kowithayakorn, T., Sakai, S., Kawahara, N., Goda, Y. Yamaguchi, N., Ishibashi, M., 2004. New chlorogenin hexasaccharide isolated from Agave fourcroydes with cytotoxic and cell cycle inhibitory activities. Bioorg. Med. Chem. 12, 3841-3845.

Oleszek, W., 1993. Allelopathic potential of alfalfa (Medicago sativa) saponins: their relation to antifungal and haemolytic activities. J. Chem. Ecol. 19, 1063-1074.

Pant, G., Sati, O.P., Miyahara, K., Kawasaki, T., 1986. Spirostanol glycosides from Agave cantala. Phytochemistry 25, 1491-1494.

Paz Mendes, T., De Medeiros Silva, G., Pereira da Silva, B., Paz Parente, J., 2004. A new steroidal saponin from Agave attenuata. Nat. Prod. Lett. 18, 183-188.

Pereira da Silva, B., Valente, A.P., Paz Parente, J., 2006a. A new steroidal saponin from Agave shrevei. Nat. Prod. Lett. 20, 385-390.

Pereira da Silva, B., Oliveira Campos, P., Paz Parente, J., 2006b. Chemical structure and biological activity of steroidal saponins from Furcraea gigantea. Chem. Nat. Comp. 42, 316-321.

Pereira da Silva, B., De Sousa, A.C., Medeiros Silva, G., Paz Mendes, T., Paz Parente, J. 2002. A new bioactive steroidal saponin from Agave attenuata. Z. Naturforsch. $57,423-428$.

Qin, X.J., Sun, D.J., Ni, W., Chen, C.X., Hua, Y., He, L., Liu, H.Y., 2012. Steroidal saponins with antimicrobial activity from stems and leaves of Paris polyphylla var. yunnanensis. Steroids 77, 1242-1248.
Sati, O.P., Pant, G., Miyahara, K., Kawasaki, T., 1985. Cantalasaponin-1, a novel spirostanol bisdesmoside from Agave cantala. J. Nat. Prod. 48, 395-399.

Sati, O.P., Rana, U., Chaukiyal, D.C., Sholichin, M., 1987. A new spirostanol glycoside from Agave cantala. J. Nat. Prod. 50, 263-265.

Scognamiglio, M., D’Abrosca, B., Fiumano, V., Chambery, A., Severino, V., Tsafantakis, N., Pacifico, S., Esposito, A., Fiorentino, A., 2012. Oleanane saponins form Bellis sylvestris Cyr. and evaluatin of their phytotoxicity on Aegilops geniculata Roth. Phytochemistry 84, 125-134.

Sharma, S.C., Sati, O.P., 1982. A spirostanol glycoside from Agave cantala. Phytochemistry 21, 1820-1821.

Sparg, S.G., Light, M.E., van Staden, J., 2004. Biological activities and distribution of plant saponins. J. Ethnopharmacol. 94, 219-243.

Tanaka, T., Nakashima, T., Ueda, T., Tomii, K., Kouno, I., 2007. Facile discrimination of aldose enantiomers by reversed-phase HPLC. Chem. Pharm. Bull. 55, 899-901.

Tinto, W.F., Simmons Boyce, J.L., McLean, S., Reynolds, W.F., 2005. Constituents of Agave americana and Agave barbadensis. Fitoterapia 76, 594-597.

Tsurumi, S., Tsujino, Y., 1995. Chromosaponin I stimulates the growth of lettuce roots. Physiol. Plant. 93, 785-789.

Uniyal, G.C., Agrawal, P.K., Thakur, R.S., Sati, O.P., 1990. Steroidal glycosides from Agave cantala. Phytochemistry 29, 937-940.

Uniyal, G.C., Agrawal, P.K., Sati, O.P., Thakur, R.S., 1991a. A spirostane hexaglycoside from Agave cantala fruits. Phytochemistry 30, 4187-4189.

Uniyal, G.C., Agrawal, P.K., Sati, O.P., Thakur, R.S., 1991b. Agaveside C, a steroidal glycoside from Agave cantala. Phytochemistry 30, 1336-1339.

Varshney, I.P., Jain, D.C., Srivastava, H.C., 1981. Cantalanin-A. A new saponin from the leaves of Agave cantala. J. Nat. Prod. 44, 662-663.

Varshney, I.P., Jain, D.C., Srivastava, H.C., 1982. A triglucoside of hecogenin from fruits of Agave cantala. Phytochemistry 21, 239-240.

Vendrig, J.C., 1964. Growth regulating activity of some saponins. Nature 203, 13011302.

Vollermer, Y.S., Gorovits, M.B., Gorovits, T.T., Abubakirov, N.K., 1978. Steroid saponins and sapogenins of Allium. XIV. Structure of karatavioside A. Khim. Prir. Soedin. 6, 740-746.

Waller, G.R., Yang, C.F., Chen, L.F., Su, C.H., Liou, R.M., Wu, S.C., Young, C.C., Lee, M.R., Lee, J.S., Chou, C.H., Kim, D., 1996. Can soyasaponin I and mono- and bidesmosides isolated from mungbeans serve as growth enhancers in mungbeans and lettuce? Adv. Exp. Med. Biol. 405, 123-139.

Yang, C.R., Zhang, Y., Jacob, M.R., Khan, S.I., Zhang, Y.J., Li, X.C., 2006. Antifungal activity of C-27 steroidal saponins. Antimicrob. Agents Chemother. 50, 17101714

Yokosuka, A., Mimaki, Y., 2007. Steroidal glycosides from Agave utahensis. Chem. Pharm. Bull. 55, 145-149.

Yokosuka, A., Mimaki, Y., 2009. Steroidal saponins from the whole plants of Agave utahensis and their cytotoxic activity. Phytochemistry 70, 807-815.

Yokosuka, A., Mimaki, Y., Kuroda, M., Sashida, Y., 2000. A new steroidal saponin from the leaves of Agave americana. Planta Med. 66, 393-395.

Zhang, Y., Zhang, Y.J., Jacob, M.R., Li, X.C., Yang, C.R., 2008. Steroidal saponins from the stem of Yucca elephantipes. Phytochemistry 69, 264-270.

Zou, P., Fu, J., Yu, H.S., Zhang, J., Kang, L.P., Ma, B.P., Yan, X.Z., 2006. The NMR studies on two new furostanol saponins from Agave sisalana leaves. Magn. Reson. Chem. 44, 1090-1095. 